\section{ÜBRIGENS}

...sind pro Jahr mehr als 41.000 Arbeitsunfähigkeits (AU)-Fälle auf Morbus Crohn zurückzuführen. 2014 lösten sie 581.268 AU-Tage aus. Wegen verminderter Erwerbstätigkeit waren 411 Rentenzugänge gezählt worden.

Weißbuch Gastroenterologische Erkrankungen

...wird M. Crohn oft mit jahrelanger Verzögerung diagnostiziert. Ähnlich verhält es sich bei der mikroskopischen Colitis, die bei einem Drittel der Patienten geradezu perfekt das Symptombild des Reizdarms imitiert.

\section{CME 2017; 6:32}

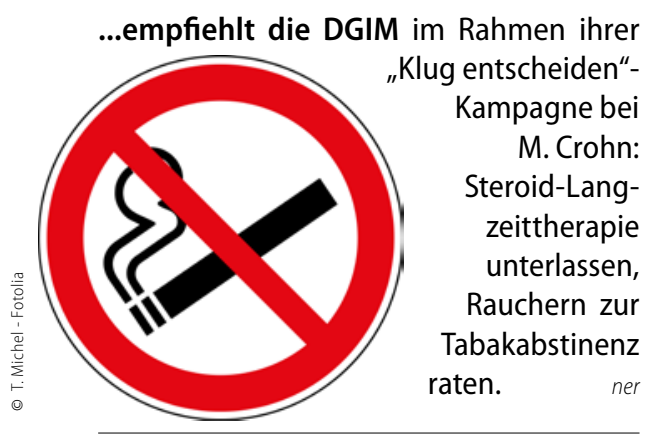

Internist 2017; 58: 539-44

\title{
Kontroverse zur Heilung der Mukosa
}

Die Mukosaheilung ist derzeit noch kein obligates Therapieziel. Aggressivität und Risiken der Therapie sollen von Ärzten und Patienten gemeinsam gegeneinander abgewogen werden.

Eine lange bestehende Entzündung führt früher oder später zu Strukturschäden. Insofern ist die „Mukosaheilung“ ein unter Gastroenterologen seit Jahren viel diskutiertes Therapieziel. Der Parameter findet sich regelhaft in klinischen Studien. Dahinter steht die Kontroverse, wie aggressiv CED-Patienten behandelt werden sollten, um langfristig einen günstigen Verlauf zu erreichen.

„Die vollständige Abheilung der Schleimhaut bei der Colitis ulcerosa wie auch beim M. Crohn stellt grundsätzlich einen klinisch günstigen Zustand für den Patienten dar", erklärt Prof. Torsten Kucharzik, Lüneburg [1]. Kucharzik ver- weist auf Beobachtungen, wonach bei jenen Patienten, bei denen eine Mukosaheilung eintritt, mit vergleichsweise günstigeren klinischen Verläufen der CED gerechnet werden kann.

\section{Was gilt für die Praxis?}

Allerdings ist unklar, ob die Mukosaheilung tatsächlich auf die jeweilige Therapie zurückzuführen ist oder ob die Patienten unter einer eher günstigen Verlaufsform der Krankheit leiden.

Im Alltag, darüber sind sich CED-Experten weitgehend einig, kommt es daher darauf an, mit den Patienten und eventuell Chirurgen gemeinsam zu besprechen, welches Therapieziel mit Blick auf die Symptomatik sowie auf den mittel- bis langfristigen Verlauf angestrebt werden soll und welche Risiken und Belastungen sie bereit sind, dafür auf sich zu nehmen.

1. MMW 2016; 158: 74-80
Punkte sammeln in der

Sommerakademie mit e.Med

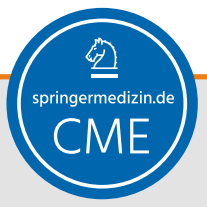

Auch 2017 haben wir in der Sommerakademie ausgewählte CME-Kurse für Ihre hausärztliche Fortbildung zusammengestellt.

Zum Thema "Morbus Crohn“ finden Sie unter

http://bit.ly/2hFwgdK

aktuell den Kurs

\section{„Aktuelle therapeutische Strategien}

\section{beim Morbus Crohn"}

Zur Teilnahme benötigen sie ein e.Med-Abo, mit dem sie Zugang zu allen Inhalten auf SpringerMedizin.de erhalten.

Nutzen Sie die Sommerzeit, um CME-Punkte zu sammeln und um sich schnell und effizient fortzubilden. Testen Sie SpringerMedizin.de 30 Tage lang kostenlos und unverbindlich mit dem e.Med-Abo: http://bit.ly/2vhm8el

\section{CED-Inzidenz}

\section{Seit Jahren erkranken mehr Kinder}

Seit Jahrzehnten erkranken immer mehr Kinder und Jugendliche an chronisch entzündlichen Darmerkrankungen (CED), besonders an Morbus Crohn.

„Am stärksten wächst die Gruppe der CED-Patienten im Alter unter zehn Jahren“, berichten Dr. Tobias Schwerd und Prof. Sibylle Koletzko von der LMU München [1]. Insgesamt erhält etwa jeder vierte CED-Patient seine Diagnose vor dem 18. Lebensjahr.

Im Vergleich zu Erwachsenen ist der intestinale Befall bei Kindern und Jugendlichen oft ausgeprägter, die Krankheit ver- schlimmert sich rascher. Die Ursachen dafür scheinen vielfältig zu sein: Beschrieben werden verschiedene genetisch bedingte Störungen, etwa Autophagie, der epithelialen Barrierefunktion, der Stressreaktion im endoplasmatischen Retikulum sowie von verschiedenen Immunantworten auf Umwelteinflüsse wie zum Beispiel Nahrungsbestandteile. "In diesem Zusammenhang ist bemerkenswert, dass Migranten, die in westliche Industrienationen ausgewandert sind, vermehrt an CED erkranken", so Schwerd und Koletzko.

1. Monatsschr Kinderhlkd 2017; 3: 257-269 